\title{
Perfectly matched layers for the convected Helmholtz. equation
}

Eliane Bécache - Anne-Sophie Bonnet-Ben Dhia — Guillaume Legendre

$$
\mathbf{N}^{\circ} 4690
$$

Janvier 2003

THÈME 4 



\title{
Perfectly matched layers for the convected Helmholtz equation
}

\author{
Eliane Bécache* , Anne-Sophie Bonnet-Ben Dhia ${ }^{\dagger}$, Guillaume Legendre $^{\ddagger}$ \\ Thème 4 - Simulation et optimisation \\ de systèmes complexes \\ Projet Ondes \\ Rapport de recherche $\mathrm{n}^{\circ} 4690$ - Janvier 2003 - 29 pages
}

\begin{abstract}
In this paper, we propose and analyze perfectly matched absorbing layers for a problem of time harmonic acoustic waves propagating in a duct in presence of a uniform flow. The absorbing layers are designed for the pressure field, satisfying the convected scalar Helmholtz equation. A difficulty, compared to the Helmholtz equation, comes from the presence of socalled inverse upstream modes which become unstable, instead of evanescent, with the classical Bérenger's PMLs. We introduce here a new PML model which makes all outgoing waves evanescent. We then analyse the error due to the truncation of the domain and prove that the convergence is exponential with respect to the size of the layers, for both the classical and the new PML models. Numerical validations are finally presented.
\end{abstract}

Key-words: acoustic waves, convected Helmholtz equation, duct modes, absorbing layers, PML, instabilities

\footnotetext{
* Institut National de Recherche en Informatique et en Automatique, Domaine de Voluceau-Rocquencourt, BP 105, 78153 Le Chesnay cedex, France (eliane.becache@inria.fr).

${ }^{\dagger}$ Laboratoire de Simulation et de Modélisation des phénomènes de Propagation, URA 853 du CNRS, ENSTA, 32 boulevard Victor, 75739 Paris cedex 15, France (bonnet@ensta.fr).

$\ddagger$ Office National d'Études et de Recherches Aérospatiales, BP 72, 29 avenue de la Division Leclerc, 92322 Châtillon cedex, France (guillaume.legendre@onera.fr).
} 


\section{Couches parfaitement adaptées pour l'équation de Helmholtz convectée}

Résumé : Nous proposons et analysons des couches absorbantes parfaitement adaptées pour un problème de propagation d'ondes acoustiques en régime harmonique dans un conduit en présence d'un écoulement uniforme. Les couches absorbantes sont construites pour le champ de pression satisfaisant l'équation de Helmholtz convectée. Une difficulté, par rapport à l'équation de Helmholtz, provient de la présence de modes dits amont inverses qui deviennent instables, au lieu d'être évanescents, avec le modèle classique de Bérenger. Nous introduisons un nouveau modèle de couches, qui rend toutes les ondes sortantes évanescentes. L'erreur due à la troncature du domaine est alors analysée et nous prouvons que la convergence est exponentielle par rapport à la taille des couches, à la fois pour le modèle classique et pour le nouveau modèle. Des validations numériques sont finalement présentées.

Mots-clés : ondes acoustiques, équation de Helmholtz convectée, modes de conduit, couches absorbantes parfaitement adaptées, instabilités 


\section{Table of Contents}

1 Introduction $\quad 3$

2 The physical and the mathematical models $\quad 5$

2.1 The problem in the infinite duct . . . . . . . . . . . . . 5

2.2 Reduction to a bounded domain . . . . . . . . . . . . . . . . . 6

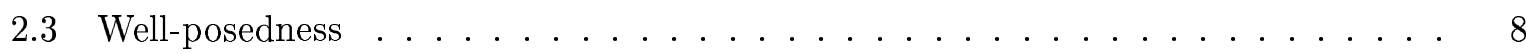

3 The Perfectly Matched Layer model 9

3.1 Modal analysis of the Bérenger's model in a waveguide . . . . . . . . . . . . 9

3.2 The new PML formulation for the convected Helmholtz equation . . . . . . . . 10

$4 \quad$ PML truncation. Error estimates $\quad 13$

4.1 Truncation of the absorbing layer and well-posedness . . . . . . . . . . . . . 13

4.2 Reduction to a problem posed in $\Omega_{b} \ldots \ldots \ldots \ldots \ldots \ldots$

4.3 Convergence and error estimates . . . . . . . . . . . . . 16

5 Varying coefficients $\quad 20$

6 Numerical results $\quad 22$

6.1 The no inverse upstream mode case . . . . . . . . . . . . . . . . . . . . 22

6.2 The inverse upstream mode case . . . . . . . . . . . . . . . . . . 24

6.3 Some practical remarks on the use of PMLs for time-harmonic problems . . . . . 26

7 Conclusion

\section{Introduction}

The Perfectly Matched Layers (PMLs) have been introduced by Bérenger [3] in order to design efficient numerical absorbing boundary conditions (more exactly absorbing layers) for the computation of time-dependent solutions of Maxwell's equations in unbounded domains. They have been applied in numerous applications since then, most in time domain $[4,28,5,23]$ but also for time-harmonic wave-like equations [27, 15].

In particular, perfectly matched layers have been used for the resolution in the time domain of the linearized Euler equations $[19,13,16,26]$, which modelize the acoustic propagation in presence of flow. In that case, it has been observed that perfectly matched layers can lead to instabilities, due to the presence of waves whose phase and group velocities have opposite signs [26] (see [2] for a general analysis of this phenomenon). Some techniques have first been developed in order to overcome this difficulty, making the layers become stable but, unfortunately, no more perfectly matched $[16,1]$. More recently, ideas for designing stable PMLs for this problem have emerged from several teams independently. These new approaches, which seem to be very closely related, have been developed for time-dependent applications in $[20,11,14]$ and for time-harmonic applications in the present paper. These different works all deal with the case of a parallel flow, which is orthogonal to the layers.

We are concerned with the propagation of acoustic waves in a duct in presence of a uniform flow. For such a mean flow, the time-harmonic linearized Euler equations reduce into a scalar convected Helmholtz equation for the pressure. Note that there is no difficulty to set the problem in a bounded domain for this particular case, thanks to the Dirichlet-to-Neumann (DtN) operator.

$\mathrm{RR} \mathrm{n}^{\circ} 4690$ 
However, the perfectly matched layers have the interest to be very simple to implement (local) compared to DtN conditions. Actually, we aim at extending this method to vectorial cases, involving more general flows. This point will be the object of a forthcoming paper.

When applying the classical (i.e. Bérenger's) PMLs to the convected Helmholtz equation in a duct, a simple modal analysis shows that the presence of the so-called inverse upstream modes produces an exponential blow-up of the solution in the space variable. This is easy to see, remembering the interpretation of the PMLs as a complex change of variable [8, 24, 10, 9]. This change of variable corresponds to a similarity applied on the axial wavenumbers of the modes. For the classical Helmholtz equation, this similarity makes all outgoing modes become evanescent. But in presence of flow, the transformation sends the inverse upstream modes into the "bad" part of the complex plane, leading to the instabilities observed in the time domain.

The idea proposed here, which is similar to the ones developed independently for time domain applications in [20,14], consists in applying a translation before the similarity to the axial wavenumbers. This removes the presence of unstable modes. It will be refered to in the following as new PMLs.

The object of this paper is the analysis of the convergence of both PML models, as the thickness of the sponge layer tends to infinity. Similar convergence analyses have already been carried out for the Helmholtz equation, via boundary integral equation techniques in [21] or the pole condition in [18]. Surprisingly, we prove that, for the convected Helmholtz equation, the two models always converge. In other words, contrary to time domain applications, the presence of unstable modes does not affect the efficiency of classical PMLs.

Finally, let us emphasize that, in most papers concerning PMLs for time-harmonic applications, coefficients are designed in order to satisfy requirements established for time domain applications. We show that this choice is too restrictive: for instance, the particular dependance of these coefficients regarding the frequency has no more justification for the present case.

The outline of this paper is as follows.

Equations of the scattering problem are presented in section 2. A formulation in a bounded domain is given, involving DtN conditions on the fictitious boundaries, which are explicited through modal expansions. Finally, the well-posedness is proven using Fredholm theory.

Classical and new PML techniques, with constant coefficients, are described in section 3: thanks to a modal analysis, these layers are sketched to be "perfectly matched". Besides, they are absorbing, except the classical PMLs in presence of the so-called inverse upstream modes.

Section 4 is devoted to the analysis of the error due to the truncation of the layers. An equivalent formulation of the problem with PMLs is written in the physical domain, the thickness of the layers appearing in the expression of the Dirichlet-to-Neumann maps. This allows to prove that both PML models converge to the physical solution, as the length of the layers tends to infinity. More precisely, the error in the physical domain does not depend on the PML model under consideration and decreases exponentially fast for both models. Note however that classical PMLs lead to an exponentially large solution in the layers, whereas the solution computed with new PMLs is evanescent in the layers.

Extension to the case of layers with spatially varying coefficients is discussed in section 5 and numerical illustrations are given in the last section. 


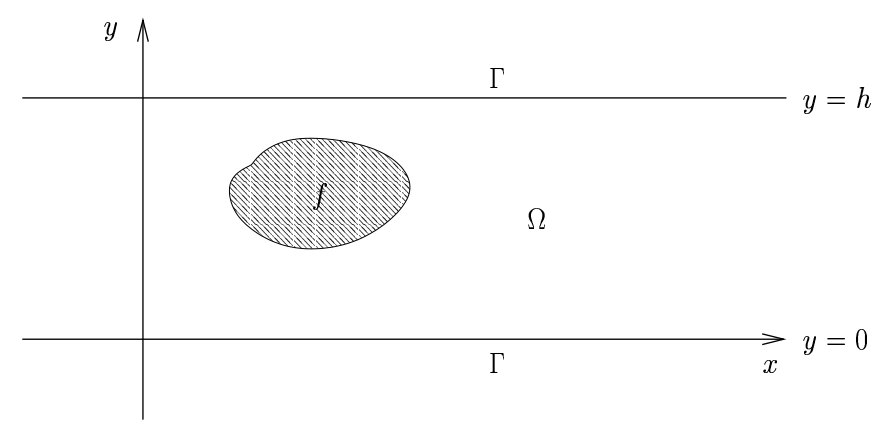

Figure 1: The infinite duct.

\section{The physical and the mathematical models}

\subsection{The problem in the infinite duct}

Let us consider the infinite rigid duct, carrying a mean fluid flow, shown in figure 1 . The problem is a two-dimensional one, set in the $x y$ plane, where the $x$ (resp. $y$ ) axis is parallel (resp. normal) to the walls of the duct. Mathematically, the duct is defined by the unbounded domain $\Omega=\mathbb{R} \times[0, h]$, where $h$ denotes the distance between the sound hard walls.

To describe the propagation of acoustic waves in the duct, we assume the following approximations to be valid:

- The fluid is homogeneous, non viscous and non heat conductive.

- The thermodynamic processes are adiabatic.

- The mean velocity $v_{0}$ is subsonic and uniform.

- The perturbations are small and equations are linear in the acoustic quantities.

- A harmonic time dependence $\exp (-i \omega t), \omega>0$ being the pulsation, is assumed (although this factor is suppressed throughout).

The acoustic pressure field $p(x, y)$ then satisfies the convected Helmholtz equation in the infinite duct:

$$
\left(1-M^{2}\right) \frac{\partial^{2} p}{\partial x^{2}}+\frac{\partial^{2} p}{\partial y^{2}}+2 i k M \frac{\partial p}{\partial x}+k^{2} p=f \text { in } \Omega
$$

where $f \in L^{2}(\Omega)$ is a compactly supported function and $M=v_{0} / c_{0}$ and $k=\omega / c_{0}$ are respectively the Mach number $(-1<M<1)$ and the wavenumber, $c_{0}$ being the sound velocity in the fluid. In addition to (1), the pressure verifies the Neumann homogeneous boundary condition on the two rigid walls of the duct $\Gamma=\partial \Omega$ :

$$
\frac{\partial p}{\partial y}=0 \text { on } \Gamma
$$

To obtain a well-posed problem, a "radiation condition", which selects the "outgoing" waves, has still to be defined at infinity. This condition is nonlocal and is expressed thanks to the Dirichletto-Neumann (DtN) operator. This requires the introduction of the so-called modes of the duct,

$\mathrm{RR} \mathrm{n}^{\circ} 4690$ 
which are the solutions of (1)-(2) in absence of source $(f=0)$ and with separated variables. These are given by:

$$
p_{n}^{ \pm}(x, y)=e^{i \beta_{n}^{ \pm} x} \varphi_{n}(y)
$$

where

$$
\varphi_{0}(y)=\sqrt{\frac{1}{h}}, \varphi_{n}(y)=\sqrt{\frac{2}{h}} \cos \left(\frac{n \pi y}{h}\right), n \in \mathbb{N}^{*},
$$

and axial wavenumbers $\beta_{n}^{ \pm}$are the solutions of

$$
-\left(1-M^{2}\right) \beta^{2}-2 k M \beta+k^{2}=\frac{n^{2} \pi^{2}}{h^{2}}, n \in \mathbb{N} .
$$

Let us introduce

$$
K_{0}=\frac{k h}{\pi \sqrt{1-M^{2}}}
$$

and let $N_{0}=\left[K_{0}\right]$ denote the floor of $K_{0}$. If $n \leq N_{0}, \beta_{n}^{ \pm}$is real and equal to

$$
\beta_{n}^{ \pm}=\frac{-k M \pm \sqrt{k^{2}-\frac{n^{2} \pi^{2}}{h^{2}}\left(1-M^{2}\right)}}{1-M^{2}} .
$$

In that case, $p_{n}^{ \pm}$is called a propagative mode. The number of propagative modes is an increasing function of the Mach number $M$, which is assumed to be positive in the remaining of the paper. Simple calculations show that the group velocity $\frac{\partial \omega}{\partial \beta}$ is positive for $p_{n}^{+}$modes and negative for $p_{n}^{-}$ones. A well-known effect of the presence of flow is the existence, when

$$
\sqrt{1-M^{2}} \frac{n \pi}{h}<k<\frac{n \pi}{h}
$$

of modes $p_{n}^{+}$which have a negative phase velocity $\frac{\omega}{\beta}$ and a positive group velocity. They are called inverse upstream modes.

The axial wavenumber $\beta_{n}^{ \pm}$is complex if $n>N_{0}$ :

$$
\beta_{n}^{ \pm}=\frac{-k M \pm i \sqrt{\frac{n^{2} \pi^{2}}{h^{2}}\left(1-M^{2}\right)-k^{2}}}{1-M^{2}} .
$$

In this case, $p_{n}^{ \pm}$is exponentially decreasing when $x \rightarrow \pm \infty$ and called an evanescent mode.

\subsection{Reduction to a bounded domain}

We now want to select the outgoing solution (1)-(2), which corresponds to superposition of $p_{n}^{+}$ (resp. $p_{n}^{-}$) modes when $x \rightarrow+\infty$ (resp. $x \rightarrow-\infty$ ), i.e. either to the propagative modes with a positive (resp. negative) group velocity or to the evanescent modes.

To derive the appropriate Dirichlet-to-Neumann boundary condition, we introduce the bounded domain $\Omega_{b}$, located in between two boundaries $\Sigma_{ \pm}$respectively located at $x=x_{-}$and $x=x_{+}$ (see figure 2), such that the support of the source $f$ is included in $\Omega_{b}$ :

$$
\Omega_{b}=\left\{(x, y) \in \Omega, x_{-} \leq x \leq x_{+}\right\}
$$




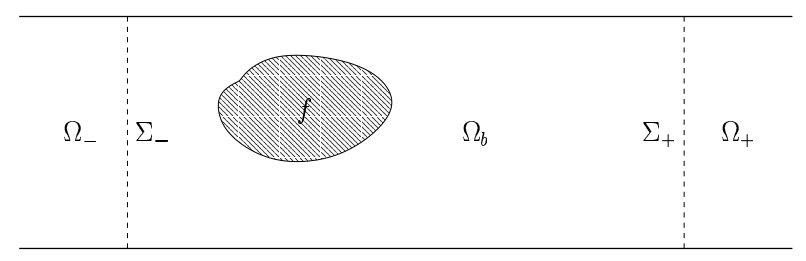

Figure 2: The bounded domain.

We set $\Omega_{ \pm}$the complementary domains

$$
\Omega_{-}=\left\{(x, y) \in \Omega, x<x_{-}\right\} \text {and } \Omega_{+}=\left\{(x, y) \in \Omega, x>x_{+}\right\} .
$$

The solution $p$ of (1) then satisfies the homogeneous equation

$$
\left(1-M^{2}\right) \frac{\partial^{2} p}{\partial x^{2}}+2 i k M \frac{\partial p}{\partial x}+\frac{\partial^{2} p}{\partial y^{2}}+k^{2} p=0 \text { in } \Omega_{ \pm},
$$

and therefore can be decomposed on the modes. Consequently, in $\Omega_{-}$, i.e. for $x<x_{-}$, we have

$$
p(x, y)=\sum_{n=0}^{+\infty}\left(p\left(x_{-}, .\right), \varphi_{n}\right)_{L^{2}\left(\Sigma_{-}\right)} \varphi_{n} e^{i \beta_{n}^{-}\left(x-x_{-}\right)},
$$

and in $\Omega_{+}$, i.e. for $x>x_{+}$,

$$
p(x, y)=\sum_{n=0}^{+\infty}\left(p\left(x_{+}, .\right), \varphi_{n}\right)_{L^{2}\left(\Sigma_{+}\right)} \varphi_{n} e^{i \beta_{n}^{+}\left(x-x_{+}\right)},
$$

where $(\cdot, \cdot)_{L^{2}\left(\Sigma_{+}\right)}\left(\operatorname{resp} .(\cdot, \cdot)_{L^{2}\left(\Sigma_{-}\right)}\right)$denotes the $L^{2}\left(\Sigma_{+}\right)$(resp. $\left.L^{2}\left(\Sigma_{-}\right)\right)$inner product for scalar functions:

$$
(u, v)_{L^{2}\left(\Sigma_{ \pm}\right)} \equiv \int_{\Sigma_{ \pm}} u(y) \bar{v}(y) \mathrm{d} y
$$

The DtN operators $T_{ \pm}$can then be defined as

$$
\begin{aligned}
T_{ \pm}: H^{1 / 2}\left(\Sigma_{ \pm}\right) & \rightarrow H^{-1 / 2}\left(\Sigma_{ \pm}\right) \\
\phi & \mapsto \mp \sum_{n=0}^{+\infty} i \beta_{n}^{ \pm}\left(\phi, \varphi_{n}\right)_{L^{2}\left(\Sigma_{ \pm}\right)} \varphi_{n}(y),
\end{aligned}
$$

and we have the following boundary conditions on $\Sigma_{ \pm}$for the solution of (1):

$$
\frac{\partial p}{\partial \boldsymbol{n}}=-T_{ \pm} p \text { on } \Sigma_{ \pm}
$$

where the vector $\boldsymbol{n}$ denotes the unit outward normal to $\Sigma_{ \pm}$.

Having established exact boundary conditions satisfied by $p$, we can now write a problem set in the bounded domain $\Omega_{b}$. More precisely, consider the following problem: find $p \in H^{1}\left(\Omega_{b}\right)$ such that

$$
\begin{cases}\left(1-M^{2}\right) \frac{\partial^{2} p}{\partial x^{2}}+2 i k M \frac{\partial p}{\partial x}+\frac{\partial^{2} p}{\partial y^{2}}+k^{2} p=f & \text { in } \Omega_{b}, \\ \frac{\partial p}{\partial y}=0 & \text { on } \Gamma \cap \partial \Omega_{b} \\ \frac{\partial p}{\partial \boldsymbol{n}}=-T_{ \pm} p & \text { on } \Sigma_{ \pm} .\end{cases}
$$

$\mathrm{RR} \mathrm{n}^{\circ} 4690$ 
The fact that $f$ is compactly supported in $\Omega_{b}$ shows clearly that problems (10) and (1)-(2) are equivalent in the sense of the following proposition:

Proposition 1 If $p$ is a solution of system (1)-(2), then $p_{\left.\right|_{\Omega_{b}}}$ is a solution of (10). Conversely, if $\widetilde{p}$ is a solution of (10), then $\widetilde{p}$ can be extended in a unique way to a solution of (1)-(2).

\subsection{Well-posedness}

Formulation (10) has two main interests. On the one hand, from a theoretical point of view, it allows to give a result of existence and uniqueness of the solution. On the other hand, we can deal numerically with the new problem, since it is set on a bounded domain.

An equivalent weak form of system (10) can then be written as follows: find $p \in H^{1}\left(\Omega_{b}\right)$ such that

$$
a_{\Omega_{b}}(p, q)=-\int_{\Omega_{b}} f \bar{q} \mathrm{~d} x \mathrm{~d} y \text { for all } q \in H^{1}\left(\Omega_{b}\right),
$$

where the sesquilinear form $a_{\Omega_{b}}(\cdot, \cdot)$ is defined by:

$$
a_{\Omega_{b}}(p, q)=b(p, q)+c(p, q)
$$

with

$$
b(p, q)=\int_{\Omega_{b}}\left(\left(1-M^{2}\right) \frac{\partial p}{\partial x} \frac{\partial \bar{q}}{\partial x}+\frac{\partial p}{\partial y} \frac{\partial \bar{q}}{\partial y}+p \bar{q}\right) \mathrm{d} x \mathrm{~d} y+\left\langle T_{+} p, q\right\rangle_{\Sigma_{+}}+\left\langle T_{-} p, q\right\rangle_{\Sigma_{-}},
$$

where the brackets $\langle\cdot, \cdot\rangle_{\Sigma_{+}}$(resp. $\langle\cdot, \cdot\rangle_{\Sigma_{-}}$) denote the natural duality pairing between $H^{-1 / 2}\left(\Sigma_{+}\right)$ and $H^{1 / 2}\left(\Sigma_{+}\right)$(resp. $H^{-1 / 2}\left(\Sigma_{-}\right)$and $\left.H^{1 / 2}\left(\Sigma_{-}\right)\right)$, and

$$
c(p, q)=\int_{\Omega_{b}}\left(-2 i k M \frac{\partial p}{\partial x} \bar{q}-\left(1+k^{2}\right) p \bar{q}\right) \mathrm{d} x \mathrm{~d} y .
$$

It is shown in [6] that this problem is of Fredholm type. By the Fredholm alternative, problem (11) is well-posed if and only if the homogeneous problem has no solution except the trivial one $p=0$.

Theorem 1 The problem is well-posed if and only if

$$
k \neq \sqrt{1-M^{2}} \frac{n \pi}{h}, \forall n \in \mathbb{N} .
$$

Proof. Suppose that $p$ is a solution of equations (1)-(2) with $f=0$. Then, there are complex constants $A_{n}^{+}$and $A_{n}^{-}$such that:

$$
p(x, y)=\sum_{n=0}^{+\infty}\left(A_{n}^{+} e^{i \beta_{n}^{+} x}+A_{n}^{-} e^{i \beta_{n}^{-} x}\right) \varphi_{n}(y),
$$

with the definitions (3) to (6). Boundary condition (9) then gives:

$$
A_{n}^{-}\left(\beta_{n}^{-}-\beta_{n}^{+}\right)=A_{n}^{+}\left(\beta_{n}^{-}-\beta_{n}^{+}\right)=0,
$$

so that $p$ vanishes identically if $\beta_{n}^{+} \neq \beta_{n}^{-}$or, likewise, if $k^{2} \neq\left(1-M^{2}\right) \frac{n^{2} \pi^{2}}{h^{2}}$.

Suppose conversely that $k=\sqrt{1-M^{2}} \frac{n \pi}{h}$, then

$$
\beta_{n}^{+}=\beta_{n}^{-}=-\frac{k M}{1-M^{2}}
$$

and $\varphi_{n}(y) e^{i \beta_{n}^{+} x}$ is a non-trivial solution of the homogeneous problem.

In the sequel, we assume the problem is well-posed, which means that (15) is satisfied. 


\section{The Perfectly Matched Layer model}

The PML model introduced by Bérenger for the time-dependent Maxwell equations can be constructed using a complex change of variable in the frequency domain as shown in $[8,10,9]$, which is the point of view adopted in the present paper. This is closely related to a theoretical approach known as dilation analyticity [17].

In this section, we briefly recall some properties of the classical PML formulation for the Helmholtz equation. Note that in the context of the propagation in a waveguide, the interpretation of the method relies on the modal approach instead of the usual plane wave one. This modal analysis allows us to point out the origin of the instabilities in the presence of flow and leads naturally to the introduction of a new model of PMLs as a remedy.

\subsection{Modal analysis of the Bérenger's model in a waveguide}

The purpose of the method is to provide a fictitious, absorbing medium, such that its interface with the "physical" bounded domain does not reflect any outgoing mode. Transposing in the frequency domain the Bérenger's formulation set in the time domain, the model consists in using the following substitution:

$$
\frac{\partial}{\partial x} \longrightarrow \alpha \frac{\partial}{\partial x}
$$

where $\alpha$ is a complex function taken as

$$
\alpha(x)=\frac{-i \omega}{-i \omega+\sigma(x)}
$$

with $\sigma(x)$ a real, positive function such as $\sigma(x)=0$ (and therefore $\alpha(x)=1$ ) in $\Omega_{b}$, the derivative with respect to $y$ being left unchanged.

In the case of the Helmholtz equation, we obtain:

$$
\alpha(x) \frac{\partial}{\partial x}\left(\alpha(x) \frac{\partial p}{\partial x}\right)+\frac{\partial^{2} p}{\partial y^{2}}+k^{2} p=f \text { in } \Omega .
$$

Note that the writing of this equation in the weak sense implies the following jump conditions at the interfaces between $\Omega_{b}$ and the PMLs:

$$
[p(x, y)]=0 \text { and }\left[\alpha(x) \frac{\partial p}{\partial x}(x, y)\right]=0 .
$$

For the modal analysis in the waveguide, we now assume that $\alpha(x)$ is a constant in $\Omega \backslash \Omega_{b}$, which we still denote by $\alpha$ for the sake of simplicity. In other words:

$$
\alpha(x)= \begin{cases}1 & \text { if } x_{+} \leq x \leq x_{-} \\ \alpha & \text { otherwise }\end{cases}
$$

For any $\alpha$, the interface between the PML and the physical domain is perfectly transparent and we will see that if $\alpha$ is well chosen, the transmitted waves decrease exponentially in the layer. Classically, the modes in a waveguide are given by

$$
p_{n}^{ \pm}(x, y)=e^{ \pm i \beta_{n} x} \varphi_{n}(y), n \in \mathbb{N}
$$

$\mathrm{RR} \mathrm{n}^{\circ} 4690$ 
where functions $\phi_{n}, \forall n \in \mathbb{N}$, are defined by (3) and axial wavenumbers $\beta_{n}$ are solutions of the dispersion relation

$$
\beta_{n}^{2}=k^{2}-\frac{n^{2} \pi^{2}}{h^{2}}, n \in \mathbb{N}
$$

such that $\beta_{n}>0$ for the propagative modes and $\operatorname{Im}\left(\beta_{n}\right)>0$ for the evanescent ones. Refering to subsection 2.1 , note that

$$
\beta_{n}=\beta_{n}^{+}=-\beta_{n}^{-},
$$

with the Mach number $M$ taken equal to zero in the definitions (5) and (6) of $\beta_{n}^{ \pm}$.

In the same manner, one can define the modes in the PML as

$$
p_{n, \alpha}^{ \pm}(x, y)=e^{ \pm i \beta_{n, \alpha} x} \varphi_{n}(y), n \in \mathbb{N}
$$

with

$$
\beta_{n, \alpha}=\frac{\beta_{n}}{\alpha}
$$

If $\alpha$ satisfies the following assumption:

$$
\operatorname{Re}(\alpha)>0, \operatorname{Im}(\alpha)<0
$$

$p_{n, \alpha}^{ \pm}$is exponentially decreasing for $x \rightarrow \pm \infty$, for any $n$. It is now straightforward to show that an incident mode $p_{n}^{+}$generates an evanescent transmitted mode $p_{n, \alpha}^{+}$in $\Omega_{+}$and no reflection at the interface $\Sigma_{+}$. Let us stress that assumption (23) is the only requirement on $\alpha$ to obtain a perfectly matched layer. Surprisingly, the more restrictive choice (17) seems to be used in most time-harmonic applications.

\subsection{The new PML formulation for the convected Helmholtz equation}

A natural idea for designing a perfectly matched layer for the convected Helmholtz equation, already used in the literature for applications in the time domain, is to apply the technique described in the previous subsection. It has been observed by several authors that this approach leads to instabilities in the time domain $[19,13,16,26]$. The presence of instabilities have been explained in [2], thanks to an analysis via group velocities. In a ducted environment, this phenomenon can be easily understood using the modal approach.

As in the no-flow case, the axial wavenumbers $\beta_{n, \alpha}^{ \pm}$of the modes $p_{n, \alpha}^{ \pm}$in the PML are given by

$$
\beta_{n, \alpha}^{ \pm}=\frac{\beta_{n}^{ \pm}}{\alpha}, n \in \mathbb{N}
$$

This can be illustrated by representing the $\beta_{n}^{ \pm}$and $\beta_{n, \alpha}^{ \pm}$in the complex plane. We clearly notice in figures 3 and 4 that the transformation

$$
\begin{aligned}
S_{\alpha}: \mathbb{C} & \rightarrow \mathbb{C} \\
z & \mapsto \frac{z}{\alpha}
\end{aligned}
$$

due to the change of variable used in the PML is a similarity of ratio $\frac{1}{\alpha \mid}$ and angle $\arg \left(\frac{1}{\alpha}\right)=$ $-\arg (\alpha)$ around the origin in the complex plane. The main difference with the Helmholtz 


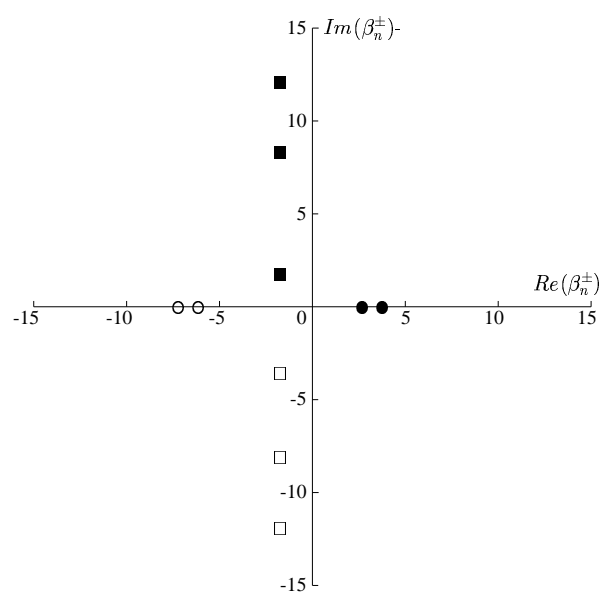

Figure 3: First axial wavenumbers of the modes for the convected Helmholtz equation $(k=$ $5, M=0.3$ and $h=1$ ). Circles and squares are respectively associated with propagative and evanescent modes, while filled and empty symbols respectively refers to downstream and upstream modes.

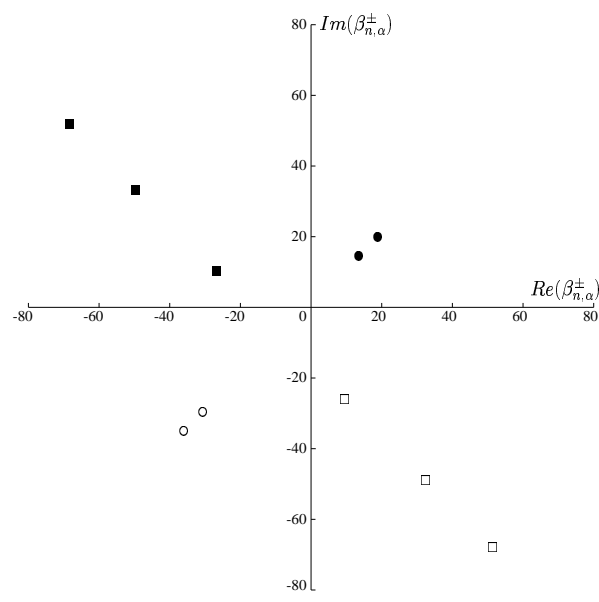

Figure 4: Effect of the similarity $S_{\alpha}(\alpha=0.1(1-i))$ on the first axial wavenumbers of the modes for the convected Helmholtz equation $(k=5, M=0.3$ and $h=1)$. 


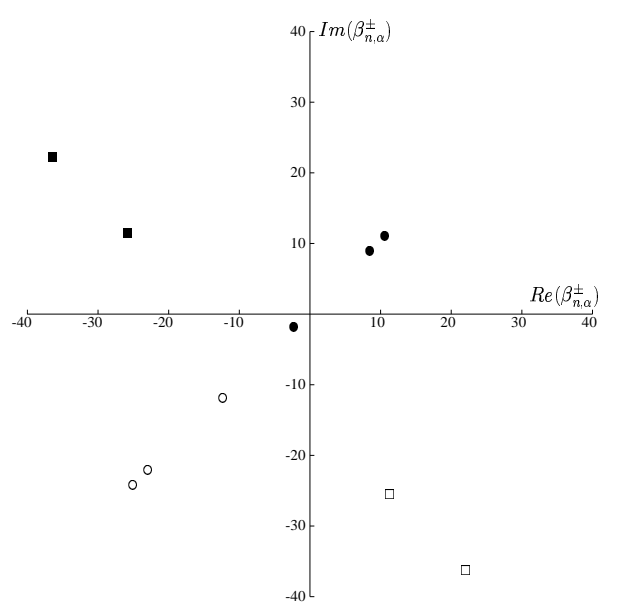

Figure 5: Effect of the similarity $S_{\alpha}(\alpha=0.2(1-i))$ on the first axial wavenumbers of the modes for the convected Helmholtz equation in presence of an inverse upstream mode $(k=6, M=0.4$ and $h=1)$.

equation is the possible existence of inverse upstream modes. Indeed, if $p_{n}^{+}$is an inverse upstream mode (as defined in subsection 2.1), the corresponding $\beta_{n}^{+}$is negative so that $\operatorname{Im}\left(\frac{\beta_{n}^{+}}{\alpha}\right)$ becomes negative for any $\alpha$ satisfying assumption (23). This is illustrated in figure 5 , the third propagative downstream mode of the presented case being an inverse upstream mode. This leads us to the conclusion that the PML model does not produce any unstable (i.e. exponentially growing in the layer) mode if all the axial wavenumbers $\beta_{n, \alpha}$ for the propagative downstream (resp. upstream) modes are strictly located in the upper (resp. lower) half of the complex plane.

Guided by the previous geometrical interpretation, we propose to apply a translation in the complex plane prior to the similarity which moves all the $\beta_{n, \alpha}^{+}$corresponding to the inverse upstream modes in the right half-plane and keeps the $\beta_{n, \alpha}^{-}$associated to propagative modes in the left one. Such a transformation is equivalent to the following substitution in equation (1):

$$
\frac{\partial}{\partial x} \longrightarrow \alpha \frac{\partial}{\partial x}+i \lambda
$$

with $\lambda \in \mathbb{R}$. The resulting axial wavenumbers are now given by

$$
\beta_{n, \alpha, \lambda}^{ \pm}=\frac{\beta_{n}^{ \pm}-\lambda}{\alpha}, n \in \mathbb{N}
$$

Although $\lambda$ could be chosen among several values, the most appropriate choice is the following:

$$
\lambda^{*}=-\frac{k M}{1-M^{2}} .
$$

This value corresponds to the real part of the wavenumbers of the evanescent modes and, for any $\alpha$ satisfying assumption (23), the $\beta_{n, \alpha, \lambda^{*}}^{ \pm}$are well located. Other choices for $\lambda$ would require further restrictions on $\alpha$ in order to ensure that $\beta_{n}$ associated with evanescent modes also stay in the good side of the complex plane (see figure 6 ).

Again, we denote in the following by $\lambda(x)$ the function defined as

$$
\lambda(x)= \begin{cases}0 & \text { if } x_{+} \leq x \leq x_{-} \\ \lambda & \text { otherwise }\end{cases}
$$




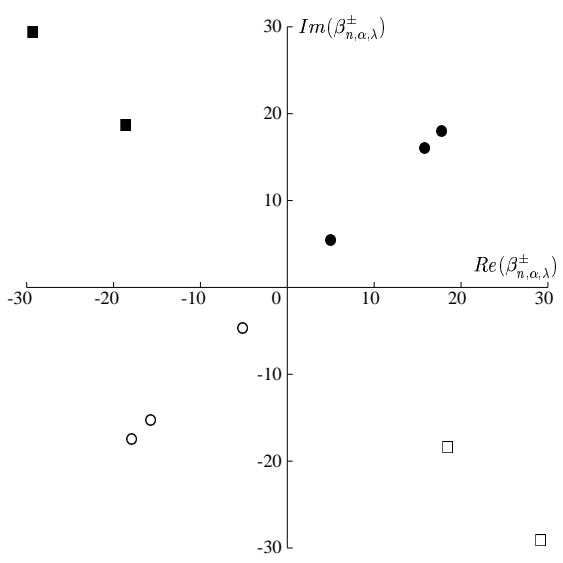

Figure 6: Effect of the new transformation (translation prior to similarity $S_{\alpha}$ ) on the first axial wavenumbers of the modes for the convected Helmholtz equation in presence of an inverse upstream mode $(k=6, M=0.4$ and $h=1), \alpha=0.2(1-i)$ and $\lambda=-\frac{k M}{1-M^{2}}$.

Finally, the equation in the new PML medium can be written as:

$$
\left(1-M^{2}\right)\left(\alpha(x) \frac{\partial}{\partial x}+i \lambda(x)\right)^{2} p+\frac{\partial^{2} p}{\partial y^{2}}+2 i k M\left(\alpha(x) \frac{\partial}{\partial x}+i \lambda(x)\right) p+k^{2} p=f \text { in } \Omega .
$$

where the function $\alpha(x)$ (resp. $\lambda(x)$ ) is defined in (20) (resp. in (26)) with $\lambda \in \mathbb{R}$ and $\alpha \in \mathbb{C}$ satisfying assumption (23). Writing this equation in a weak sense implies jump conditions at the interfaces between $\Omega_{b}$ and the layers:

$$
[p(x, y)]=0 \text { and }\left[\alpha(x) \frac{\partial p}{\partial x}(x, y)+i \lambda(x) p(x, y)\right]=0 .
$$

Remark: This new change of variable can also be used to derive stable PMLs in the time domain, as done in $[20,14]$.

\section{PML truncation. Error estimates}

\subsection{Truncation of the absorbing layer and well-posedness}

Until now, we have considered an absorbing layer of infinite length. In practice, one has to bound the computational domain and layers are of finite length $L$ in this section.

We denote by $\Omega^{L}$ the truncated domain and by $\Sigma_{ \pm}^{L}$ the external boundaries, presented in figure 7 . For simplicity, we choose to use homogeneous Dirichlet boundary conditions on these boundaries,

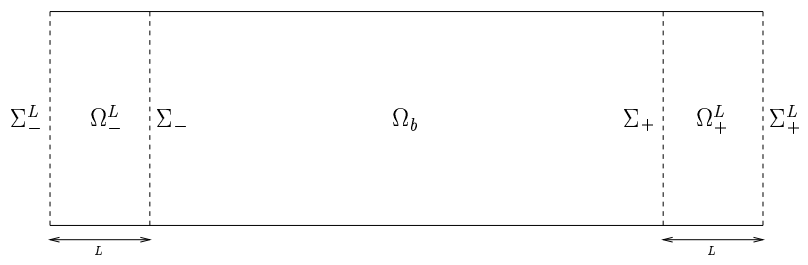

Figure 7: The truncated domain $\Omega^{L}$.

$\mathrm{RR} \mathrm{n}^{\circ} 4690$ 
but the analysis done in the following would still be valid for the natural boundary conditions $\alpha \frac{\partial p}{\partial x}+i \lambda p=0$. Let $p^{L}$ denote the solution in the truncated domain, satisfying

$$
\begin{cases}\left(1-M^{2}\right)\left(\alpha(x) \frac{\partial}{\partial x}+i \lambda(x)\right)^{2} p+\frac{\partial^{2} p}{\partial y^{2}} & \\ +2 i k M\left(\alpha(x) \frac{\partial}{\partial x}+i \lambda(x)\right) p+k^{2} p=f & \text { in } \Omega^{L}, \\ \frac{\partial p^{L}}{\partial y}=0 & \text { on } \Gamma \cap \partial \Omega^{L}, \\ p^{L}=0 & \text { on } \Sigma_{ \pm}^{L} .\end{cases}
$$

Denoting $V_{L}=\left\{q \in H^{1}\left(\Omega^{L}\right) \mid q=0\right.$ on $\left.\Sigma_{ \pm}^{L}\right\}$, a variational formulation of (29) can be written as follows: find $p^{L} \in V_{L}$ such that

$$
a_{\Omega^{L}}\left(p^{L}, q\right)=-\int_{\Omega^{L}} \frac{1}{\alpha} f \bar{q} \mathrm{~d} x \mathrm{~d} y, \forall q \in V_{L},
$$

where the sesquilinear form $a_{\Omega^{L}}(\cdot, \cdot)$ is defined as

$$
a_{\Omega^{L}}(p, q)=b_{L}(p, q)+c_{L}(p, q),
$$

with

$$
b_{L}(p, q)=\int_{\Omega^{L}}\left(\left(1-M^{2}\right) \alpha \frac{\partial p}{\partial x} \frac{\partial \bar{q}}{\partial x}+\frac{1}{\alpha} \frac{\partial p}{\partial y} \frac{\partial \bar{q}}{\partial y}+p \bar{q}\right) \mathrm{d} x \mathrm{~d} y
$$

and

$$
\begin{aligned}
c_{L}(p, q)= & \int_{\Omega^{L}} i\left(\left(\left(M^{2}-1\right) \lambda-2 k M\right) \frac{\partial p}{\partial x} \bar{q}+\left(1-M^{2}\right) \lambda p \frac{\partial \bar{q}}{\partial x}\right) \mathrm{d} x \mathrm{~d} y \\
& +\int_{\Omega^{L}}\left(\left(1-M^{2}\right) \frac{\lambda^{2}}{\alpha}+2 k M \frac{\lambda}{\alpha}-\frac{k^{2}}{\alpha}-1\right) p \bar{q} \mathrm{~d} x \mathrm{~d} y .
\end{aligned}
$$

Theorem 2 If $\alpha$ satisfies (23), problem (30) is of Fredholm type.

Proof. The bounded operator $C_{L}$ on $H^{1}\left(\Omega^{L}\right)$, defined by the Riesz representation theorem as

$$
\left(C_{L} p, q\right)_{H^{1}\left(\Omega^{L}\right)}=c_{L}(p, q), \forall(p, q) \in H^{1}\left(\Omega^{L}\right)^{2},
$$

is clearly compact (from the compacity of the embedding of $H^{1}\left(\Omega^{L}\right)$ into $L^{2}\left(\Omega^{L}\right)$ ). On the other hand, the sesquilinear form $b_{L}(\cdot, \cdot)$ is coercive on $V_{L}$. To check this, it suffices to take the real part of $b_{L}(q, q)$ :

$$
\begin{aligned}
\operatorname{Re}\left(b_{L}(q, q)\right) & =\int_{\Omega^{L}}\left(\operatorname{Re}(\alpha)\left(1-M^{2}\right)\left|\frac{\partial q}{\partial x}\right|^{2}+R e\left(\frac{1}{\alpha}\right)\left|\frac{\partial q}{\partial y}\right|^{2}+|q|^{2}\right) \mathrm{d} x \mathrm{~d} y \\
& \geq C\|q\|_{H^{1}\left(\Omega^{L}\right)}^{2}
\end{aligned}
$$

where, thanks to assumption (23), $C$ is a strictly positive constant depending on complex constant $\alpha$ and Mach number $M$ :

$$
C=\inf \left(\left(1-M^{2}\right) \operatorname{Re}(\alpha), \operatorname{Re}\left(\frac{1}{\alpha}\right), 1\right)
$$

INRIA 


\subsection{Reduction to a problem posed in $\Omega_{b}$}

Remember that our original problem (1)-(2) has been proved in section 2.2 to be equivalent to the problem (10) posed in $\Omega_{b}$. Having in mind the comparison of the solution $p^{L}$ of the problem (29), posed in the truncated domain, with the solution $p$ of the original problem, we first reformulate (29) as a problem posed only in $\Omega_{b}$. Consider the following problem: find $p_{b}^{L} \in H^{1}\left(\Omega_{b}\right)$ such that

$$
\begin{cases}\left(1-M^{2}\right) \frac{\partial^{2} p_{b}^{L}}{\partial x^{2}}+2 i k M \frac{\partial p_{b}^{L}}{\partial x}+\frac{\partial^{2} p_{b}^{L}}{\partial y^{2}}+k^{2} p_{b}^{L}=f & \text { in } \Omega_{b} \\ \frac{\partial p_{b}^{L}}{\partial y}=0 & \text { on } \Gamma \cap \partial \Omega_{b} \\ \frac{\partial p_{b}^{L}}{\partial \boldsymbol{n}}=-T_{ \pm}^{L} p_{b}^{L} & \text { on } \Sigma_{ \pm}\end{cases}
$$

where $T_{ \pm}^{L}$ are operators defined as follows:

$$
\begin{aligned}
T_{ \pm}^{L}: H^{1 / 2}\left(\Sigma_{ \pm}\right) & \rightarrow H^{-1 / 2}\left(\Sigma_{ \pm}\right) \\
\phi & \mapsto \mp \sum_{n=0}^{+\infty} i \nu_{n}^{ \pm}(L)\left(\phi, \varphi_{n}\right)_{L^{2}\left(\Sigma_{ \pm}\right)} \varphi_{n}(y)
\end{aligned}
$$

with

$$
\nu_{n}^{ \pm}(L)=\beta_{n}^{ \pm}+\frac{\beta_{n}^{\mp}-\beta_{n}^{ \pm}}{1-e^{i\left(\beta_{n}^{\mp}-\beta_{n}^{ \pm}\right) L / \alpha}} .
$$

Note that values $\nu_{n}^{ \pm}(L)$ are well defined, thanks to assumption (15).

Proposition 2 If $p^{L}$ is a solution of (29), then $p_{\left.\right|_{\Omega_{b}}}$ is a solution of (31). Conversely, if $p_{b}^{L}$ is a solution of (31), then it can be extended in a unique way to a solution of (29).

Proof. The key idea to reformulate the problem as a problem posed in $\Omega_{b}$ is to write an exact boundary condition satisfied by the solution on the boundaries $\Sigma_{ \pm}$. We set $\Omega_{ \pm}^{L}$ the complementary domains:

$$
\Omega_{-}^{L}=\left\{(x, y) \in \Omega^{L}, x_{-} L<x<x_{-}\right\} \text {and } \Omega_{+}^{L}=\left\{(x, y) \in \Omega^{L}, x_{+}<x<x_{+}+L\right\} .
$$

Since $p_{ \pm}^{L}=p_{\left.\right|_{\Omega_{ \pm}^{L}} ^{L}}^{L}$ satisfies a homogeneous equation in these domains, it can be explicited through a modal expansion. Consider for instance the solution in the right domain $\Omega_{+}^{L}$. Using the Dirichlet boundary condition on the external layer boundary $\Sigma_{+}^{L}$, the solution can be written as

$$
p_{+}^{L}(x, y)=\sum_{n=0}^{+\infty}\left(p_{+}^{L}\left(x_{+}, .\right), \varphi_{n}\right)_{L^{2}\left(\Sigma_{+}\right)}\left(A_{n}^{+} e^{i \gamma_{n}^{+}\left(x-x_{+}\right)}+A_{n}^{-} e^{i \gamma_{n}^{-}\left(x-x_{+}\right)}\right) \varphi_{n}(y),
$$

where we have denoted $\gamma_{n}^{ \pm}=\beta_{n, \alpha, \lambda}^{ \pm}$, for the sake of clarity, and

$$
A_{n}^{ \pm}=\mp \frac{e^{i \gamma_{n}^{\mp} L}}{e^{i \gamma_{n}^{+} L}-e^{i \gamma_{n}^{-} L}} .
$$

We check easily that these quantities are always defined. Actually, the denominator would vanish if there existed an integer $n$ for which $\left(\gamma_{n}^{+}-\gamma_{n}^{-}\right) L \in 2 \pi \mathbb{Z}$, which means that $\left(\beta_{n}^{+}-\beta_{n}^{-}\right) L / \alpha \in 2 \pi \mathbb{Z}$.

RR $\mathrm{n}^{\circ} 4690$ 
If $k^{2} \neq\left(1-M^{2}\right) \frac{n^{2} \pi^{2}}{h^{2}}$, the quantity $\beta_{n}^{+}-\beta_{n}^{-}$is never zero. Furthermore, with $\alpha$ satisfying assumption (23), $\left(\beta_{n}^{+}-\beta_{n}^{-}\right) L / \alpha$ has always a non-zero imaginary part and thus cannot belong to $2 \pi \mathbb{Z}$. We then write an exact boundary condition satisfied by $p_{+}^{L}$ on $\Sigma_{+}$:

$$
\left(\frac{\partial p_{+}^{L}}{\partial x}\right)_{\left.\right|_{\Sigma_{+}}}=\sum_{n=0}^{+\infty}\left(p_{+}^{L}\left(x_{+}, .\right), \varphi_{n}\right)_{L^{2}\left(\Sigma_{+}\right)}\left(A_{n}^{+} i \gamma_{n}^{+}+A_{n}^{-} i \gamma_{n}^{-}\right) \varphi_{n}(y) .
$$

Using the jump conditions (28) and relation (24), this yields an exact boundary condition satisfied in the interior by $p_{b}^{L}$ :

$$
\begin{aligned}
\left(\frac{\partial p_{b}^{L}}{\partial x}\right)_{\left.\right|_{\Sigma_{+}}} & =\alpha\left(\frac{\partial p_{+}^{L}}{\partial x}\right)_{\left.\right|_{\Sigma_{+}}}+i \lambda p_{+\left.\right|_{\Sigma_{+}}}^{L} \\
& \left.=i \sum_{n=0}^{+\infty}\left(p_{b}^{L}\left(x_{+}, .\right), \varphi_{n}\right)_{L^{2}\left(\Sigma_{+}\right)}\left(A_{n}^{+} \beta_{n}^{+}+A_{n}^{-} \beta_{n}^{-}\right)\right) \varphi_{n}(y) .
\end{aligned}
$$

Setting $\nu_{n}^{+}(L)=A_{n}^{+} \beta_{n}^{+}+A_{n}^{-} \beta_{n}^{-}$, this can also be written as:

$$
\left(\frac{\partial p_{b}^{L}}{\partial x}\right)_{\left.\right|_{\Sigma_{+}}}=-T_{+}^{L}\left(p_{b}^{L}\right)_{\Sigma_{\Sigma_{+}}}
$$

where $T_{+}^{L}$ denotes the operator defined in (32).

Remark: It clearly appears in the expression (33) that operators $T_{ \pm}^{L}$, and thus problem (31), do not depend on $\lambda$. In fact, the computed solution $p^{L}$ depends on $\lambda$ only in the layers.

\subsection{Convergence and error estimates}

We have shown that original system (1)-(2) and system (29) with absorbing layers of finite length are both equivalent to problems posed only in $\Omega_{b}$, respectively (10) and (31). We are now able to compare the solutions of these two problems, which are solutions of the following variational formulations:

For the original problem (10): find $p_{b} \in H^{1}\left(\Omega_{b}\right)$ such that

$$
a_{\Omega_{b}}\left(p_{b}, q\right)=-\int_{\Omega_{b}} f \bar{q} \mathrm{~d} x \mathrm{~d} y, \forall q \in H^{1}\left(\Omega_{b}\right)
$$

where the sesquilinear form $a_{\Omega_{b}}(\cdot, \cdot)$ is given $(12)$ and can be written as

$$
a_{\Omega_{b}}(p, q)=(A p, q)_{H^{1}\left(\Omega_{b}\right)}+\left\langle T_{+} p, q\right\rangle_{\Sigma_{+}}+\left\langle T_{-} p, q\right\rangle_{\Sigma_{-}}
$$

with $A$ the bounded linear operator on $H^{1}\left(\Omega_{b}\right)$ defined by

$$
(A p, q)_{H^{1}\left(\Omega_{b}\right)}=\int_{\Omega_{b}}\left(\left(1-M^{2}\right) \frac{\partial p}{\partial x} \frac{\partial \bar{q}}{\partial x}+\frac{\partial p}{\partial y} \frac{\partial \bar{q}}{\partial y}-2 i k M \frac{\partial p}{\partial x} \bar{q}-k^{2} p \bar{q}\right) \mathrm{d} x \mathrm{~d} y .
$$

For the problem with finite length absorbing layers (31): find $p_{b}^{L} \in H^{1}\left(\Omega_{b}\right)$ such that

$$
a_{\Omega_{b}}^{L}\left(p_{b}^{L}, q\right)=-\int_{\Omega_{b}} f \bar{q} \mathrm{~d} x \mathrm{~d} y, \forall q \in H^{1}\left(\Omega_{b}\right)
$$


where the sesquilinear form $a_{\Omega_{b}}^{L}(\cdot, \cdot)$ can be written as

$$
a_{\Omega_{b}}^{L}(p, q)=(A p, q)_{H^{1}\left(\Omega_{b}\right)}+\left\langle T_{+}^{L} p, q\right\rangle_{\Sigma_{+}}+\left\langle T_{-}^{L} p, q\right\rangle_{\Sigma_{-}},
$$

the operator $A$ being defined in (36).

To prove the convergence and get error estimates, we follow an idea developed in [25], which has also been used in [12]. We first show the

Lemma 1 Suppose that assumptions (15) and (23) hold. Then, there exist strictly positive constants $\mathcal{C}=\mathcal{C}(k, M)$ and $\eta=\eta(\theta, k, h, M)$ (where $\theta$ denotes the argument of $\alpha$ ) such that, for all $(p, q) \in\left(H^{1}\left(\Omega_{b}\right)\right)^{2}$, we have:

$$
\left|a_{\Omega_{b}}(p, q)-a_{\Omega_{b}}^{L}(p, q)\right| \leq \mathcal{C} e^{-\eta L /|\alpha|}\|p\|_{H^{1}\left(\Omega_{b}\right)}\|q\|_{H^{1}\left(\Omega_{b}\right)} .
$$

More precisely, the constant $\eta$ is determined by:

$$
\eta=\frac{2 k}{1-M^{2}} \min \left(-\sin (\theta) \sqrt{1-\frac{N_{0}^{2}}{K_{0}{ }^{2}}}, \cos (\theta) \sqrt{\frac{\left(N_{0}+1\right)^{2}}{K_{0}{ }^{2}}-1}\right),
$$

where $K_{0}$ is defined in (4).

Proof. From expressions (35) and (38), we have

$$
a_{\Omega_{b}}(p, q)-a_{\Omega_{b}}^{L}(p, q)=\left(\left\langle T_{+} p, q\right\rangle_{\Sigma_{+}}-\left\langle T_{+}^{L} p, q\right\rangle_{\Sigma_{+}}\right)+\left(\left\langle T_{-} p, q\right\rangle_{\Sigma_{-}}-\left\langle T_{-}^{L} p, q\right\rangle_{\Sigma_{-}}\right) .
$$

Let us focus on the first term in the right hand side, the estimation of the second one being analogous. From the definitions (8) and (32) of operators $T_{+}$and $T_{+}^{L}$, we have, for any $\phi \in$ $H^{1 / 2}\left(\Sigma_{+}\right)$,

$$
\left(T_{+}-T_{+}^{L}\right) \phi=-\sum_{n=0}^{+\infty} i\left(\beta_{n}^{+}-\nu_{n}^{+}(L)\right) \phi_{n} \varphi_{n}(y) \text { with } \phi_{n}=\left(\phi, \varphi_{n}\right)_{L^{2}\left(\Sigma_{+}\right)} .
$$

Therefore, for any $(\phi, \psi) \in\left(H^{1 / 2}\left(\Sigma_{+}\right)\right)^{2}$,

$$
\left\langle\left(T_{+}-T_{+}^{L}\right) \phi, \psi\right\rangle_{\Sigma_{+}}=-\sum_{n=0}^{+\infty} i\left(\beta_{n}^{+}-\nu_{n}^{+}(L)\right) \phi_{n} \bar{\psi}_{n}
$$

with $\phi_{n}=\left(\phi, \varphi_{n}\right)_{L^{2}\left(\Sigma_{+}\right)}$and $\psi_{n}=\left(\psi, \varphi_{n}\right)_{L^{2}\left(\Sigma_{+}\right)}$. This implies the following estimate:

$$
\left|\left\langle\left(T_{+}-T_{+}^{L}\right) \phi, \psi\right\rangle_{\Sigma_{+}}\right| \leq \sum_{n=0}^{+\infty}\left|\beta_{n}^{+}-\nu_{n}^{+}(L)\right|\left|\phi_{n} \bar{\psi}_{n}\right| .
$$

From (33), we have

$$
\left|\beta_{n}^{+}-\nu_{n}^{+}(L)\right|=\frac{\left|\beta_{n}^{+}-\beta_{n}^{-}\right|}{\left|1-e^{i\left(\beta_{n}^{-}-\beta_{n}^{+}\right) L / \alpha}\right|} .
$$

Noticing that, for any $z \in \mathbb{C}$,

$$
\left|1-e^{i z}\right| \geq\left|e^{-\operatorname{Im}(z)}-1\right|
$$

$\mathrm{RR} \mathrm{n}^{\circ} 4690$ 
so that, if $\operatorname{Im}(z)<0$ and $|\operatorname{Im}(z)|$ is large enough, this quantity is larger than

$$
\left|1-e^{i z}\right| \geq\left|e^{-\operatorname{Im}(z)}-1\right| \geq \frac{1}{2} e^{-\operatorname{Im}(z)} .
$$

We can easily check, thanks to assumption (23), that, for all $n \in \mathbb{N}$, we have $\operatorname{Im}\left(\left(\beta_{n}^{-}-\beta_{n}^{+}\right) L / \alpha\right)<$ 0 , so that, for $L$ large enough, the previous estimate gives us

$$
\left|\beta_{n}^{+}-\nu_{n}^{+}(L)\right| \leq 2\left|\beta_{n}^{+}-\beta_{n}^{-}\right| e^{\operatorname{Im}\left(\left(\beta_{n}^{-}-\beta_{n}^{+}\right) L / \alpha\right)} .
$$

Let us now distinguish the two cases:

The propagative modes $n \leq N_{0}$ From (5), we have

$$
\beta_{n}^{+}-\beta_{n}^{-}=\frac{2 k}{1-M^{2}} \sqrt{1-\frac{n^{2}}{K_{0}^{2}}}=\delta_{n}>0
$$

Noticing that

$$
\delta_{N_{0}} \leq \delta_{n} \leq \frac{2 k}{1-M^{2}}
$$

estimate (42) yields

$$
\left|\beta_{n}^{+}-\nu_{n}^{+}(L)\right| \leq 2 \delta_{n} e^{-\delta_{N_{0}} L \operatorname{Im}(1 / \alpha)} \leq \frac{4 k}{1-M^{2}} e^{-\delta_{N_{0}} L \operatorname{Im}(1 / \alpha)} .
$$

The evanescent modes $n \geq N_{0}+1 \quad$ From (6), we have

$$
\beta_{n}^{+}-\beta_{n}^{-}=\frac{2 i k}{1-M^{2}} \sqrt{\frac{n^{2}}{K_{0}^{2}}-1}=i \delta_{n}, \delta_{n}>0 .
$$

This time, $\delta_{n}$ is increasing and $\sqrt{\frac{n^{2}}{K_{0}{ }^{2}}-1} \leq \frac{n}{K_{0}}$. Estimate (42) thus yields

$$
\left|\beta_{n}^{+}-\nu_{n}^{+}(L)\right| \leq \frac{4 k}{1-M^{2}} \frac{n}{K_{0}} e^{-\delta_{N_{0}+1} L R e(1 / \alpha)}
$$

By substituting these estimates in (41), we see that

$$
\begin{aligned}
\left|\left\langle\left(T_{+}-T_{+}^{L}\right) \phi, \psi\right\rangle_{\Sigma_{+}}\right| \leq \frac{4 k}{1-M^{2}} & \left(\sum_{n=0}^{N_{0}} e^{-\delta_{N_{0}} L \operatorname{Im}(1 / \alpha)}\left|\phi_{n} \bar{\psi}_{n}\right|\right. \\
& \left.+\sum_{n=N_{0}+1}^{+\infty} \frac{n}{K_{0}} e^{-\delta_{N_{0}+1} L \operatorname{Re}(1 / \alpha)}\left|\phi_{n} \bar{\psi}_{n}\right|\right) .
\end{aligned}
$$

Setting $\eta=|\alpha| \min \left(\delta_{N_{0}} \operatorname{Im}(1 / \alpha), \delta_{N_{0}+1} \operatorname{Re}(1 / \alpha)\right)$, we then have

$$
\begin{aligned}
\left|\left\langle\left(T_{+}-T_{+}^{L}\right) \phi, \psi\right\rangle_{\Sigma_{+}}\right| & \leq \frac{4 k}{1-M^{2}} e^{-\eta L /|\alpha|} \sum_{n=0}^{+\infty}\left(1+\frac{n^{2}}{K_{0}^{2}}\right)^{1 / 2}\left|\phi_{n} \bar{\psi}_{n}\right| \\
& \leq \mathcal{C} e^{-\eta L /|\alpha|}|\phi|_{H^{1 / 2}\left(\Sigma_{+}\right)}|\psi|_{H^{1 / 2}\left(\Sigma_{+}\right)}
\end{aligned}
$$


The trace theorem finally yields, for any $(p, q) \in\left(H^{1}\left(\Omega_{b}\right)\right)^{2}$,

$$
\left|\left\langle\left(T_{+}-T_{+}^{L}\right) p, q\right\rangle_{\Sigma_{+}}\right| \leq \mathcal{C} e^{-\eta L /|\alpha|}\|p\|_{H^{1}\left(\Omega_{b}\right)}\|q\|_{H^{1}\left(\Omega_{b}\right)} .
$$

One can obviously obtain the same estimate on $\Sigma_{-}$and finally prove claim (39).

Setting $V=H^{1}\left(\Omega_{b}\right)$, we introduce linear operators $\mathcal{A}$ and $\mathcal{A}^{L}$ in $\mathcal{L}\left(V, V^{\prime}\right)$, respectively associated to the sesquilinear forms $a_{\Omega_{b}}(\cdot, \cdot)$ and $a_{\Omega_{b}}^{L}(\cdot, \cdot): \forall(p, q) \in V^{2}$,

$$
\langle\mathcal{A} p, q\rangle_{V^{\prime}, V}=a_{\Omega_{b}}(p, q) \text { and }\left\langle\mathcal{A}^{L} p, q\right\rangle_{V^{\prime}, V}=a_{\Omega_{b}}^{L}(p, q) .
$$

Obviously estimate (39) implies

$$
\left\|\mathcal{A}-\mathcal{A}^{L}\right\|_{\mathcal{L}\left(V, V^{\prime}\right)} \leq \mathcal{C} e^{-\eta L /|\alpha|}
$$

Problems (34) and (37) can both be written in terms of these operators as

$$
\begin{aligned}
\mathcal{A} p_{b} & =-f, \\
\mathcal{A}^{L} p_{b}^{L} & =-f .
\end{aligned}
$$

It follows from taking the difference between (46) and (47) that the error $p_{b}-p_{b}^{L}$ satisfies the following equation:

$$
\mathcal{A}^{L}\left(p_{b}-p_{b}^{L}\right)=\left(\mathcal{A}^{L}-\mathcal{A}\right) p_{b}
$$

Thanks to estimate (45), we are now able to show the following result:

Theorem 3 Suppose that assumptions (23) and (15) hold. There exists $L_{1}>0$ such that for all $L \geq L_{1}, \mathcal{A}^{L}$ is an isomorphism on $H^{1}\left(\Omega_{b}\right)$ and the solution $p_{b}^{L}$ of problem (31) converges to the solution $p_{b}$ of problem (10). Furthermore, there exists a constant $\mathcal{C}$ depending on $M$ and $k$ such that:

$$
\left\|p_{b}-p_{b}^{L}\right\|_{V} \leq \mathcal{C} e^{-\eta L /|\alpha|}\left\|p_{b}\right\|_{V}
$$

with $\eta$ being defined in (40).

Proof. For $g \in V^{\prime}$, we consider the problem: find $u \in V$ such that

$$
\mathcal{A}^{L} u=g \text {. }
$$

We can rewrite the operator $\mathcal{A}^{L}$ as $\mathcal{A}^{L}=\mathcal{A}+\left(\mathcal{A}^{L}-\mathcal{A}\right)$ and, using that $\mathcal{A}$ is an isomorphism on $V$,

$$
\mathcal{A}^{L}=\mathcal{A}\left(I+\mathcal{A}^{-1}\left(\mathcal{A}^{L}-\mathcal{A}\right)\right)
$$

Problem (50) thus becomes

$$
\left(I+\mathcal{A}^{-1}\left(\mathcal{A}^{L}-\mathcal{A}\right)\right) u=\mathcal{A}^{-1} g .
$$

Applying the Banach fixed point theorem, this problem admits a unique solution if

$$
\left\|\mathcal{A}^{-1}\left(\mathcal{A}^{L}-\mathcal{A}\right)\right\|_{\mathcal{L}\left(V, V^{\prime}\right)}<1
$$

$\mathrm{RR} \mathrm{n}^{\circ} 4690$ 
which is satisfied as soon as

$$
\left\|\mathcal{A}^{L}-\mathcal{A}\right\|_{\mathcal{L}\left(V, V^{\prime}\right)}<\left\|\mathcal{A}^{-1}\right\|_{\mathcal{L}\left(V, V^{\prime}\right)}^{-1}
$$

This can be achieved for $L$ large enough, $L \geq L_{1}$, since $\left\|\mathcal{A}^{L}-\mathcal{A}\right\|_{\mathcal{L}\left(V, V^{\prime}\right)}$ tends to zero as $L$ tends to infinity, thanks to (45). Moreover, we have:

$$
\left\|\left(I+\mathcal{A}^{-1}\left(\mathcal{A}^{L}-\mathcal{A}\right)\right)^{-1}\right\|_{\mathcal{L}\left(V, V^{\prime}\right)}<\frac{1}{1-\left\|\mathcal{A}^{-1}\left(\mathcal{A}^{L}-\mathcal{A}\right)\right\|_{\mathcal{L}\left(V, V^{\prime}\right)}},
$$

which implies the following estimate:

$$
\|u\|_{V}<\frac{\left\|\mathcal{A}^{-1} g\right\|_{V}}{1-\left\|\mathcal{A}^{-1}\left(\mathcal{A}^{L}-\mathcal{A}\right)\right\|_{\mathcal{L}\left(V, V^{\prime}\right)}} .
$$

Applying this result to the error, solution of problem (48) yields

$$
\begin{aligned}
\left\|p_{b}-p_{b}^{L}\right\|_{V} & <\frac{\left\|\mathcal{A}^{-1}\left(\mathcal{A}^{L}-\mathcal{A}\right) p_{b}\right\|_{V}}{1-\left\|\mathcal{A}^{-1}\left(\mathcal{A}^{L}-\mathcal{A}\right)\right\|_{\mathcal{L}\left(V, V^{\prime}\right)}} \\
& \leq \frac{\left\|\mathcal{A}^{-1}\right\|_{\mathcal{L}\left(V, V^{\prime}\right)}\left\|\mathcal{A}^{L}-\mathcal{A}\right\|_{\mathcal{L}\left(V, V^{\prime}\right)}\left\|p_{b}\right\|_{V}}{\left.1-\left\|\mathcal{A}^{-1}\right\|_{\mathcal{L}\left(V, V^{\prime}\right)} \| \mathcal{A}^{L}-\mathcal{A}\right) \|_{\mathcal{L}\left(V, V^{\prime}\right)}}
\end{aligned}
$$

When $\left\|\mathcal{A}^{L}-\mathcal{A}\right\|_{\mathcal{L}\left(V, V^{\prime}\right)}$ is small enough, the quantity in the right hand side can be bounded by

$$
\left\|p_{b}-p_{b}^{L}\right\|_{V} \leq 2\left\|\mathcal{A}^{-1}\right\|_{\mathcal{L}\left(V, V^{\prime}\right)}\left\|\mathcal{A}^{L}-\mathcal{A}\right\|_{\mathcal{L}\left(V, V^{\prime}\right)}\left\|p_{b}\right\|_{V} \leq 2 \mathcal{C} e^{-\eta}\left\|p_{b}\right\|_{V}
$$

the last inequality coming from (45).

Remarks: 1. We emphasize that error estimate (49) of theorem 3 does not depend on parameter $\lambda$. As a consequence, exponential convergence is obtained for both the classical and the new PML models. This is a main difference of behaviour from the classical PMLs in the time domain, as in that case the layers lead to instabilities in presence of inverse upstream modes $[19,26,1,2]$.

2. Note that estimate (49) also proves that convergence holds when the length of the layers $L$ is fixed and $|\alpha|$ tends to 0 . This is useful in practice for numerical computations. Indeed, $L$ has to be small in order to reduce the number of degrees of freedom. Moreover, it is more convenient to change the value of parameter $\alpha$ than the length of the layers, which requires a new mesh of the computational domain.

3. The value of $\eta$ is strongly related to the position of wavenumber $k$ with respect to the cut-off frequencies. More precisely, for a given value of the argument $\theta$ of coefficient $\alpha$, the accuracy deteriorates when $k$ is close to a cut-off wavenumber (see figure 8 ).

\section{$5 \quad$ Varying coefficients}

In practical computations, it is very common to use a spatially varying coefficient $\alpha(x)$ in the layers. Actually, it has been proven for finite difference schemes that discontinuities of $\alpha$ through the boundaries $\Sigma_{ \pm}$generate spurious reflections after discretization [10]. In this section, we show that the analysis done previously for constant coefficients $\alpha$ and $\lambda$ can be easily extended to varying coefficients. Let us point out however that the numerical results presented in the 


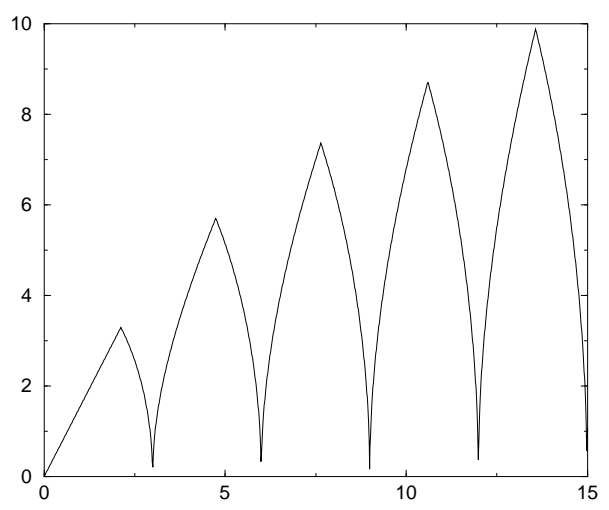

Figure 8: Coefficient $\eta$ plotted as a function of wavenumber $k$ for $M=0.3, h=1$ and $\theta=-\frac{\pi}{4}$.

next section are obtained with constant coefficients and that no significant effects due to the discontinuities have been observed.

Let $\alpha$ and $\lambda$ be two functions of coordinate $x$, defined from $\mathbb{R}$ to $\mathbb{C}$, such that:

$$
\alpha(x)=1 \text { and } \lambda(x)=0 \text { for } x \in\left[x_{-}, x_{+}\right] .
$$

We assume moreover that $\alpha(x)$ satisfies (23) for all $x>x_{+}$or $x<x_{-}$. Let us consider once more problem (29). Since the proof of theorem 2 does not use the fact that $\alpha$ and $\lambda$ are constant in the layers, the theorem still holds and the problem is of Fredholm type.

To establish a convergence result with respect to the size of the layers, we follow the steps of sections 4.2 and 4.3. The main point is that modal solutions in the layers are now

$$
p_{n}^{ \pm}(x, y)=\psi_{n}^{ \pm}(x) \varphi_{n}(y), n \in \mathbb{N},
$$

where $\varphi_{n}$ is given by (3) and $\psi_{n}^{ \pm}$is defined by:

$$
\left\{\begin{array}{l}
\left(\alpha(x) \frac{d}{d x}+i \lambda(x)\right) \psi_{n}^{ \pm}=i \beta_{n}^{ \pm} \psi_{n}^{ \pm} \\
\psi_{n}^{ \pm}\left(x_{ \pm}\right)=1 .
\end{array}\right.
$$

It then follows from straightforward calculations that problem (29) is equivalent to problem (31) set in domain $\Omega_{b}$, with the following new definition for coefficients $\nu_{n}^{ \pm}(L)$ :

$$
\nu_{n}^{ \pm}(L)=\beta_{n}^{ \pm}+\frac{\beta_{n}^{\mp}-\beta_{n}^{ \pm}}{1-\frac{\psi_{n}^{\mp}\left(x_{ \pm}+L\right)}{\psi_{n}^{ \pm}\left(x_{ \pm}+L\right)}} .
$$

Note moreover that

$$
\frac{\psi_{n}^{-}\left(x_{+}+L\right)}{\psi_{n}^{+}\left(x_{+}+L\right)}=e^{i\left(\beta_{n}^{-}-\beta_{n}^{+}\right)} \int_{x_{+}}^{x_{+}+L} \frac{1}{\alpha(x)} \mathrm{d} x,
$$

so that again $\nu_{n}^{ \pm}(L)$ do not depend on $\lambda$.

The final result then reads:

$\mathrm{RR} \mathrm{n}^{\circ} 4690$ 
Theorem 4 Problem (29) is well-posed and the solution $p_{b}^{L}$ of problem (31), with $\nu_{n}^{ \pm}(L)$ defined by (51), exists and converges to the solution $p_{b}$ of problem (10) as $L \rightarrow+\infty$. Furthermore, there exist three constants $\mathcal{C}=\mathcal{C}(k, M)$ and $\tau_{ \pm}=\tau_{ \pm}(k, M, \alpha, L)$ such that:

$$
\left\|p_{b}-p_{b}^{L}\right\|_{V} \leq \mathcal{C}\left(e^{-\tau_{+}}+e^{-\tau_{-}}\right)\left\|p_{b}\right\|_{V}
$$

with

$$
\tau_{ \pm}=\frac{2 k}{1-M^{2}} \min \left(\operatorname{Im}\left(I_{ \pm}\right) \sqrt{1-\frac{N_{0}^{2}}{K_{0}^{2}}}, \operatorname{Re}\left(I_{ \pm}\right) \sqrt{\frac{\left(N_{0}+1\right)^{2}}{K_{0}^{2}}-1}\right)
$$

where $I \pm= \pm \int_{x_{ \pm}}^{x_{ \pm} \pm L} \frac{1}{\alpha(x)} \mathrm{d} x, K_{0}$ is defined in (4) and $N_{0}$ denotes the floor of $K_{0}$.

\section{$6 \quad$ Numerical results}

In order to illustrate the conclusions previously laid on the PML models, numerical examples are presented. The following configuration is considered: the computational domain is the same as the one presented in figure 7, extending from $x=-0.2$ to $x=2.2$ and $y=0$ to $y=1$. The layers occupy the region from $x=-0.2$ to $x=0$ in the downstream direction and from $x=2$ to $x=2.2$ in the upstream direction, the thickness $L$ of the layers then being fixed and equal to $10 \%$ of the length of domain $\Omega_{b}$. A compactly supported source $f$ is given by:

$$
f=\left\{\begin{array}{l}
1 \text { if }(x-1)^{2}+(y-0.7)^{2} \leq 0.04 \\
0 \text { elsewhere }
\end{array}\right.
$$

The numerical solution of problem (30) posed in the domain bounded with PML is compared to the computed solution (which is called the reference solution) of problem (11) posed in the domain bounded with Dirichlet-to-Neumann operators. Both approximations are done with a finite element method. The DtN map, usually expressed through an infinite series expansion, is here approximated by truncating the series.

All the simulations have been conducted with the same unstructured mesh, whose mesh size is linked to the problem via a resolution of approximately 20 nodal points per wavelength when using second-order triangular $\left(P_{2}\right)$ Lagrange finite elements. For the computation of the reference solution, the number of terms in the truncated DtN map is 8, which is sufficient for accuracy in every of the tested cases. The coefficient $\alpha$ is chosen to be a complex constant in the layers, whose argument is taken equal to $-\frac{\pi}{4}$, and coefficient $\lambda$ of the new PML model takes the value $\lambda^{*}$ defined in (25). Homogeneous Dirichlet boundary conditions are imposed on the outer boundaries of the layers. The computations are done with the finite element library MÉLinA [22].

\subsection{The no inverse upstream mode case}

In this first simulation, we choose $k=10$ and $M=0.3$. For such values of wavenumber and Mach number, 4 modes are propagative and there is no inverse upstream mode. The solution in the layers is then exponentially decaying for both the classical (i.e. $\lambda=0$ ) and new PML models.

In figure 9 , the relative error to the reference solution in $H^{1}\left(\Omega_{b}\right)$ norm is plotted as a function of the modulus of $\alpha$ for the two models. We observe no noticeable discrepancy between the classical and new PML models, which behave similarly in this case. Both curves present a minimum plateau and we can roughly distinguish three zones, as indicated in figure 9: 


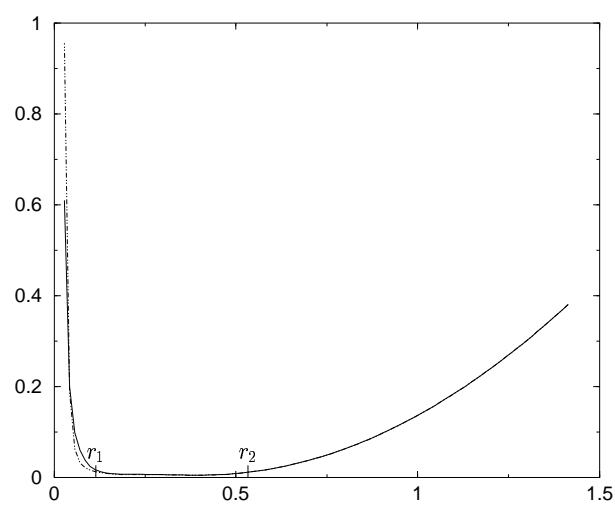

Figure 9: Relative error $\frac{\left\|p-p_{\text {ref }}\right\|_{H^{1}\left(\Omega_{b}\right)}}{\left\|p_{\text {ref }}\right\|_{H^{1}\left(\Omega_{b}\right)}}$ as a function of $|\alpha|, k=14$ and $M=0.2$. Solid line is the result for the new PML model while the dotted line refers to the classical PML model.

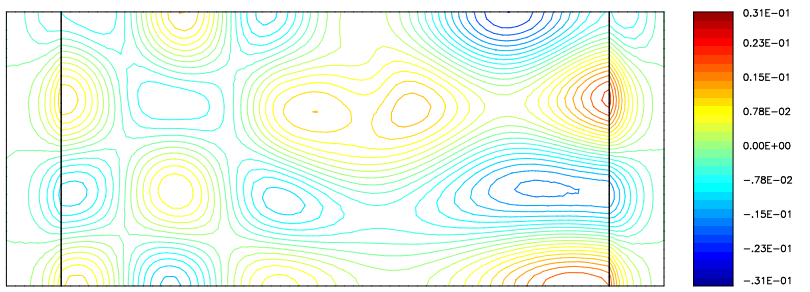

Figure 10: Real part of the pressure field, $k=10$ and $M=0.3, \alpha=.28(1-i)$ and $\lambda=-\frac{k M}{1-M^{2}}$.

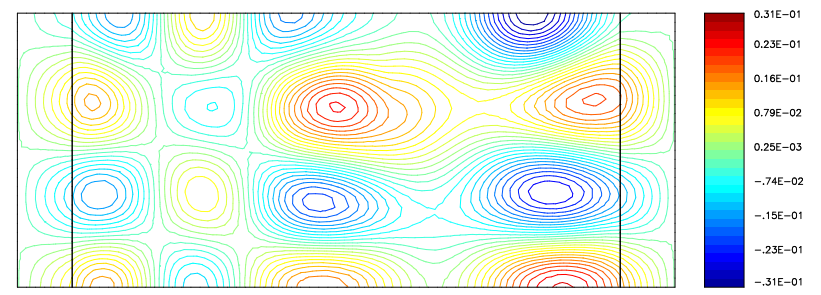

Figure 11: Real part of the pressure field, $k=10$ and $M=0.3, \alpha=1-i$ and $\lambda=-\frac{k M}{1-M^{2}}$.

- $|\boldsymbol{\alpha}| \in\left[\boldsymbol{r}_{\mathbf{1}}, \boldsymbol{r}_{\mathbf{2}}\right]$ A very good agreement between the DtN and the PML solutions, for the classical and new PML models, is obtained for a large range of values of $|\alpha|$ corresponding to the plateau seen in figure 9. The real part of the corresponding solution is represented in figure 10. We also observe in this figure the effect of the flow on the propagation of sound, as the wavelength of the solution is longer downstream of the source than upstream.

$\bullet|\boldsymbol{\alpha}|>\boldsymbol{r}_{\mathbf{2}}$ For larger values of $|\alpha|$, the layer is insufficiently absorbing and a reflection occurs at the end of the layers, as represented in figure 11.

$\bullet|\boldsymbol{\alpha}|<\boldsymbol{r}_{\mathbf{1}}$ For small values of $|\alpha|$, the absorption in the layers is high but the mesh resolution becomes too coarse to correctly represent modes in the PML medium, thus producing spurious numerical errors as seen in figure 12.

We want to confirm the convergence estimate of theorem 3, which implies:

$$
-\ln \left(\frac{\left\|p_{b}-p_{b}^{L}\right\|_{V}}{\left\|p_{b}\right\|_{V}}\right) \geq \frac{\eta L}{|\alpha|}-\ln (\mathcal{C}) .
$$

$\mathrm{RR} \mathrm{n}^{\circ} 4690$ 


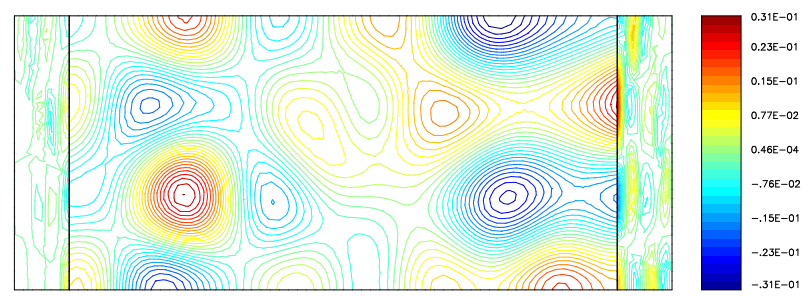

Figure 12: Real part of the pressure field, $k=10$ and $M=0.3, \alpha=.02(1-i)$ and $\lambda=-\frac{k M}{1-M^{2}}$.

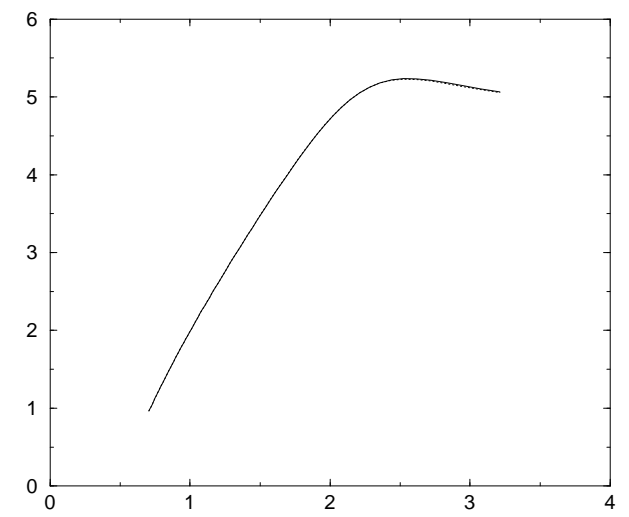

Figure 13: $-\ln \left(\frac{\left\|p-p_{\text {ref }}\right\|_{H^{1}\left(\Omega_{b}\right)}}{\left\|p_{\text {ref }}\right\|_{H^{1}\left(\Omega_{b}\right)}}\right)$ as a function of $\frac{1}{\alpha \alpha}, k=10$ and $M=0.3$. Solid and dotted lines respectively refer to results for the new and classical PML models.

To this end, the opposite of the logarithm of the relative error in $H^{1}\left(\Omega_{b}\right)$ norm is plotted as a function of the inverse of $|\alpha|$ for both PML models. The exponential convergence of the method, which can be deduced from the slope of curves in figure 13, agrees satisfactorily with the estimation given by the theory for both PML models, as the two curves coincide for this case.

\subsection{The inverse upstream mode case}

For the choice of $k=9$ and $M=0.4$, the last of the four propagative downstream modes (i.e. $n=3$ ) has a negative phase velocity and is therefore called an inverse upstream mode. The solution in the downstream layer is then exponentially decreasing or increasing with the distance, depending on the applied PML model. Results for this case are shown in figures 14 and 15 , where the relative error in $H^{1}\left(\Omega_{b}\right)$ norm and the opposite of its logarithm are respectively represented.

As one can observe in the zoom in figure 14, curves of the relative error present again a minimum plateau for both PML models. This time, the size of the plateau is smaller for the classical PML and the error for this model has a rather erratic behaviour for small values of $|\alpha|$. The convergence of the method is nonetheless achieved for both models, with the predicted exponential rate (see figure 15). However, the new PML model seems better suited to practical computations, as one can choose an appropriate and optimal value of $\alpha$ for convergence more conveniently.

At last, figures 16 and 17 respectively show the solutions for the new and classical models, the value of $|\alpha|$ for this case corresponding to the minimum of the curves in figure 14 . Note 

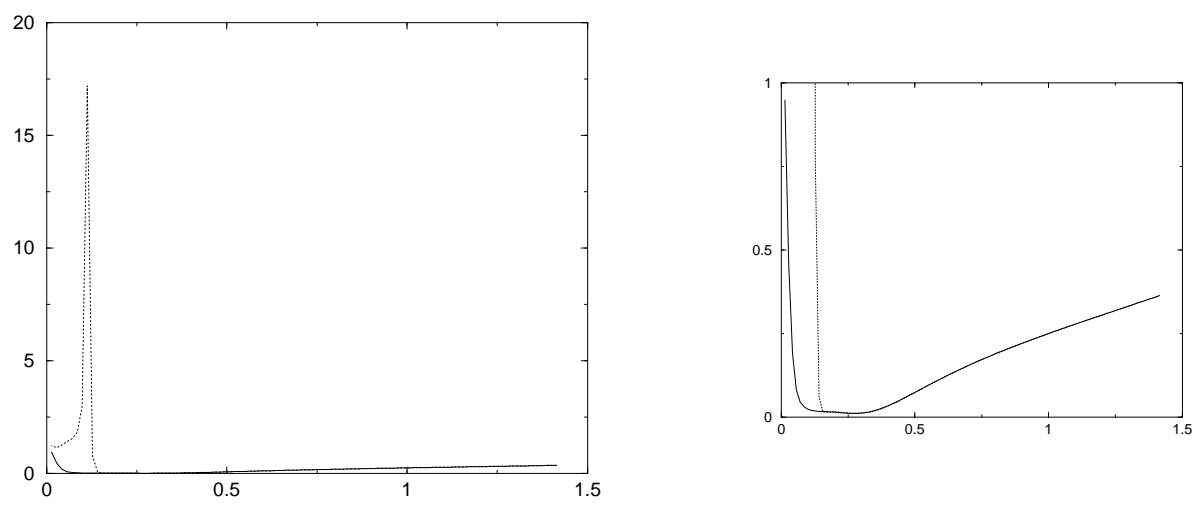

Figure 14: Left: relative error $\frac{\left\|p-p_{\text {ref }}\right\|_{H^{1}\left(\Omega_{b}\right)}}{\left\|p_{\text {ref }}\right\|_{H^{1}\left(\Omega_{b}\right)}}$ as a function of $|\alpha|, k=9$ and $M=0.4$, right: zoom on the zone of interest. Solid line is the result for the new PML model while the dotted line refers to the classical PML model.

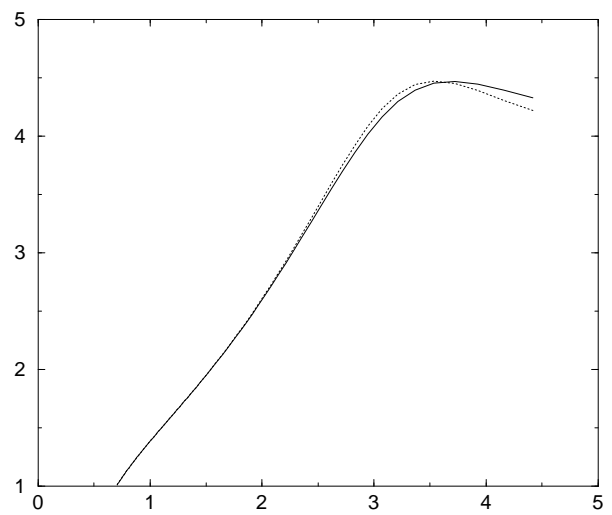

Figure 15: $-\ln \left(\frac{\left\|p-p_{\text {ref }}\right\|_{H^{1}\left(\Omega_{b}\right)}}{\left\|p_{\text {ref }}\right\|_{H^{1}\left(\Omega_{b}\right)}}\right)$ as a function of $\frac{1}{\alpha \mid}, k=9$ and $M=0.4$. Solid and dotted lines respectively refer to results for the new and classical PML models. 


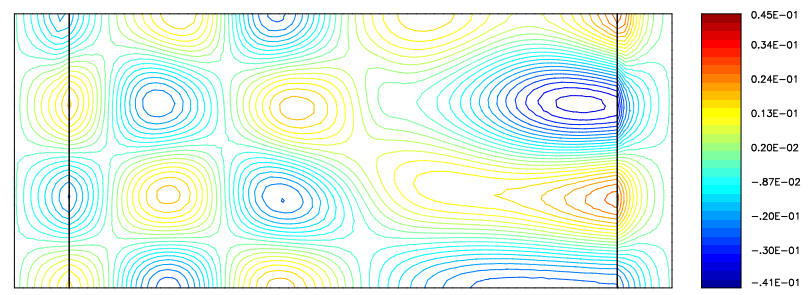

Figure 16: Real part of the pressure field, $k=9$ and $M=0.4, \alpha=.19(1-i)$ and $\lambda=-\frac{k M}{1-M^{2}}$.

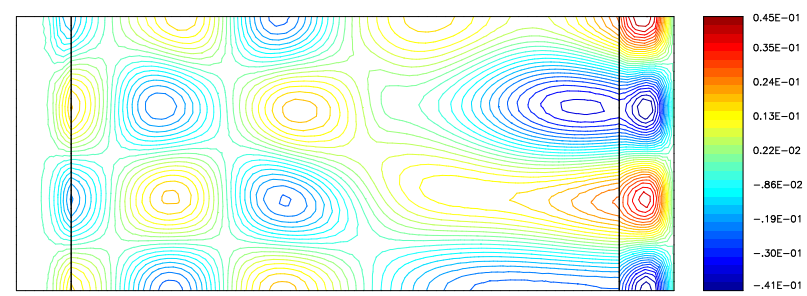

Figure 17: Real part of the pressure field, $k=9$ and $M=0.4, \alpha=.19(1-i)$ and $\lambda=0$.

that whatever the behaviour of the solution in the layers, the solution in the "physical" domain remains almost the same.

\subsection{Some practical remarks on the use of PMLs for time-harmonic problems}

We would like to point out that the numerical analysis which has been carried out in this section was based on the knowledge of a reference solution. In practice, it would be useful to have $a$ posteriori criteria which indicate if the numerical solution is satisfactory or not. In transient applications, the quality of the PML model is ensured as soon as the reflections produced at the interface between the physical and the absorbing layer can be neglected. In particular, if the excitation is a pulse localized in time, the exact solution should vanish after a large time, which gives a criterion to evaluate the efficiency of the absorbing layer. The situation is completely different in time-harmonic applications. For instance:

- The notion of reflection is more difficult to exploit: as illustrated in the previous numerical results, it is not obvious to distinguish a "reflected" wave from an "incident" wave.

- The experiment of a pulse localized in time has no counterpart in time harmonic applications.

- A good choice of the absorbing layer parameters allows to select the outgoing solution of the problem. A bad choice $(\operatorname{Im}(\alpha)>0)$ would select the ingoing solution, which is difficult to detect when one does not know the exact solution.

- However, one can notice that when $|\alpha|$ is too small, spurious numerical errors are observed. They can be removed by refining the mesh inside the layer.

\section{Conclusion}

In this paper, we have studied perfectly matched layers for the convected Helmholtz equation. In presence of inverse upstream modes, the solution can have arbitrary large values in the classical PMLs, thus causing the instabilities observed in time domain applications. We have proposed a 
new PML model which always leads to an exponentially decreasing solution in the layer, even in presence of inverse upstream modes. The error analysis surprisingly showed the convergence for both the classical and new models. Nevertheless, numerical results seem to indicate that the error is better controlled with the new model when inverse upstream modes are present. In order to understand the different numerical behaviours of the two models, it remains to analyse the convergence of the solution of the discretized PML models with respect to both the finite element mesh size and the layer parameters $\alpha$ and $L$.

This is a preliminary step in dealing with more complex time-harmonic problems. In particular, it would be interesting to extend the present method to non uniform flows. This gives raise to several difficulties. First, even for a parallel flow, the problem cannot be reduced to a simple scalar equation anymore and has to be modelized with a vectorial model, for instance linearized Euler equations or Galbrun's equation [7]. Furthermore, a modal analysis cannot be done so easily, since the orthogonality of the modes is lost and their completeness is an open question. Finally, for some flows, there exist physical outgoing unstable modes which have to be adequately treated by the absorbing model.

\section{References}

[1] S. Abarbanel, D. Gottlieb, And J. S. Hesthaven, Well-Posed perfectly matched layers for advective acoustics, J. Comput. Phys., 154 (1999), pp. 266-283.

[2] E. BéCache, S. Fauqueux, And P. Joly, Stability of perfectly matched layers, group velocities and anisotropic waves, to appear (2003).

[3] J.-P. BÉRENGER, A perfectly matched layer for the absorption of electromagnetic waves, J. Comput. Phys., 114 (1994), pp. 185-200.

[4] - Three-dimensional perfectly matched layer for the absorption of electromagnetic waves, J. Comput. Phys., 127 (1996), pp. 363-379.

[5] — Improved PML for the FDTD solution of wave-structure interaction problems, IEEE Trans. Antennas and Propagation, 45 (1997), pp. 466-473.

[6] A.-S. Bonnet-Ben Dhia, L. Dahi, E. Lunéville, and V. Pagneux, Acoustic diffraction by a plate in a uniform flow, Math. Models Methods Appl. Sci., 12 (2002), pp. 625-647.

[7] A.-S. Bonnet-Ben Dhia, G. Legendre, and E. Lunéville, Analyse mathématique de l'équation de Galbrun en écoulement uniforme, C. R. Acad. Sci. Paris Sér. IIb Méc., 329 (2001), pp. 601-606.

[8] W. C. Chew And W. H. Weedon, A 3D perfectly matched medium from modified Maxwell's equations with stretched coordinates, IEEE Microwave Opt. Technol. Lett., 7 (1994), pp. 599-604.

[9] F. Collino And P. Monk, The perfectly matched layer in curvilinear coordinates, SIAM J. Sci. Comput., 19 (1998), pp. 2061-2090.

[10] F. Collino And P. B. Monk, Optimizing the perfectly matched layer, Comput. Methods Appl. Mech. Engrg., 164 (1998), pp. 157-171.

[11] J. DiAZ AND P. Joly, Stabilized perfectly matched layers for advective wave equations, in preparation.

$\mathrm{RR} \mathrm{n}^{\circ} 4690$ 
[12] D. Gómez Pedreira And P. Joly, A method for computing guided waves in integrated optics. Part II: numerical approximation and error analysis, SIAM J. Numer. Anal., 39 (2002), pp. 1684-1711.

[13] J. W. Goodrich And T. Hagstrom, A comparison of two accurate boundary treatments for computational aeroacoustics, AIAA Paper, 97-1585 (1997).

[14] T. Hagstrom and I. Nazarov, Absorbing layers and radiation boundary conditions for jet flow simulations, in Proc. of the 8th AIAA/CEAS aeroacoustics conference, 17-19 june 2002, Breckenridge, CO, USA. AIAA paper 2002-2606.

[15] I. Harari, M. Slavutin, And E. Turkel, Analytical and numerical studies of a finite element PML for the Helmholtz equation, J. Comput. Acoust., 8 (2000), pp. 121-137.

[16] J. S. Hesthaven, On the analysis and construction of perfectly matched layers for the linearized Euler equations, J. Comput. Phys., 142 (1998), pp. 129-147.

[17] P. D. Hislop AND I. M. Sigal, Introduction to spectral theory with applications to Schrödinger operators, vol. 113 of Applied Mathematical Sciences, Springer-Verlag, NewYork, 1996.

[18] T. Hohage, F. Schmidt, And L. Zschiedrich, Solving time-harmonic scattering problems based on the pole condition: convergence of the PML method, Tech. Report 01-23, Konrad-Zuse-Zentrum für Informationstechnik Berlin, 2001.

[19] F. Q. Hu, On absorbing boundary conditions for linearized Euler equations by a perfectly matched layer, J. Comput. Phys., 129 (1996), pp. 201-219.

$[20]$ — A stable, perfectly matched layer for linearized Euler equations in unsplit physical variables, J. Comput. Phys., 173 (2001), pp. 455-480.

[21] M. Lassas And E. Somersalo, On the existence and convergence of the solution of PML equations, Computing, 60 (1998), pp. 229-241.

[22] D. Martin, On line documentation of MÉLina, http://www.maths.univrennes1.fr/dmartin/melina/www/homepage.html.

[23] P. G. Petropoulos, Reflectionless sponge layers as absorbing boundary condition for the numerical solution of Maxwell's equations in rectangular, cylindrical, and spherical coordinates., SIAM J. Appl. Math., 60 (2000), pp. 1037-1058.

[24] C. M. RAPPAPORT, Perfectly matched absorbing conditions based on anisotropic lossy mapping of space, IEEE Microwave Guided Wave Lett., 5 (1995), pp. 90-92.

[25] J. RaZAfiarivelo, Optimisation de formes en électromagnétisme, PhD thesis, Université de Paris VI, 1996.

[26] C. K. W. Tam, L. Auriault, and F. Cambuli, Perfectly matched layer as an absorbing boundary condition for the linearized Euler equations in open and ducted domains, J. Comput. Phys., 144 (1998), pp. 213-234.

[27] E. TuRkel And A. Yefet, Absorbing PML boundary layers for wave-like equations, Appl. Numer. Math., 27 (1998), pp. 533-557. 
[28] L. Zhao And A. C. CAngellaris, GT-PML: Generalized theory of perfectly matched layers and its application to the reflectionless truncation of finite-difference time-domain grids, IEEE Trans. Microwave Theory Tech., 44 (1996), pp. 2555-2563.

$\mathrm{RR} \mathrm{n}^{\circ} 4690$ 


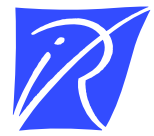

Unité de recherche INRIA Rocquencourt Domaine de Voluceau - Rocquencourt - BP 105 - 78153 Le Chesnay Cedex (France)

Unité de recherche INRIA Lorraine : LORIA, Technopôle de Nancy-Brabois - Campus scientifique 615, rue du Jardin Botanique - BP 101 - 54602 Villers-lès-Nancy Cedex (France)

Unité de recherche INRIA Rennes : IRISA, Campus universitaire de Beaulieu - 35042 Rennes Cedex (France)

Unité de recherche INRIA Rhône-Alpes : 655, avenue de l'Europe - 38330 Montbonnot-St-Martin (France)

Unité de recherche INRIA Sophia Antipolis : 2004, route des Lucioles - BP 93 - 06902 Sophia Antipolis Cedex (France)

INRIA - Domaine de Voluceau - Rocquencourt, BP 105 - 78153 Le Chesnay Cedex (France)

http://www.inria.fr

ISSN 0249-6399 